\title{
Ovonic Materials for Memory Nano-Devices: Stability of $I(V)$ Measurements
}

\author{
Massimo Rudan, Enrico Piccinini \\ ARCES and Department DEI, University of Bologna \\ Viale Risorgimento 2, I-40136 Bologna, Italy \\ Email: massimo.rudan@unibo.it
}

\author{
Fabrizio Buscemi, Rossella Brunetti \\ Department of Physics, Informatics, and Mathematics \\ University of Modena and Reggio Emilia \\ Via Campi 213/A, I-41125, Modena, Italy
}

\begin{abstract}
Measurements of the $I(V)$ characteristic in Ovonic semiconductors are notoriously unstable. Experimental setups must therefore rely on pulsed schemes in which only the positiveslope branches of the characteristic are detected. This paper considers the time-dependent, trap-limited conduction model proposed by the authors for investigating this type of devices, and shows that the model is suitable for stability analysis. The conditions that make the measurement stable are assessed; also, examples of simulations in the oscillatory regime are given, in the field-driven case.
\end{abstract}

\section{INTRODUCTION}

The electrical switching observed in a class of amorphous semiconductors, referred to as "Ovonic", is due to a transition process from a high- to a low-resistivity state occurring when a threshold field is reached [1]-[15]. The $I(V)$ characteristic shows a negative differential-resistance region above the threshold point. Among the several interpretations of the Ovonic switching in steady-state conditions, a basic analytical model relying on a thermally-assisted trap-limited conduction scheme has recently been shown to be able to reproduce the above mentioned electrical bistability [16]. The model has then been extended to the dynamic case [17]. The investigations on the device models have strongly been stimulated by the technological exploitation in the design of phase-change memories [12]. Such applications require not only predictive tools for the threshold switching in steadystate conditions, but also the analysis of the electric behavior of Ovonic materials in the dynamic and transient regime. It is well known that instability degrades the quality of the $I(V)$ curve measurement, so that only the positive-slope branches may actually be traced. This paper addresses the latter issue, showing that the model of [17] is amenable to extracting the measurement-stabilizing conditions.

\section{MODEL}

The starting point is the time-dependent model of [17]. It reads

$$
\begin{gathered}
J=q \mu n_{B} F, \\
J F=n k \frac{T_{e}-T_{0}}{\tau_{R}}+\Delta E \frac{\mathrm{d}}{\mathrm{d} t}\left[n_{B}\left(T_{e}\right)-n_{B}\left(T_{0}\right)\right], \\
\frac{n}{n_{B}}=1+c \exp \left(\frac{\Delta E}{k T_{e}}\right),
\end{gathered}
$$

ARCES is the acronym for "E. De Castro" Advanced Research Center on Electronic Systems of the University of Bologna. where $J, q, \mu$, and $F$ are the current density, electronic charge, mobility of the band electrons, and electric field, respectively. As the sample under investigation is assumed one dimensional and uniform, the equations bear no dependence on position. In turn, $n, n_{B}$ are the concentrations of all electrons and of band electrons, respectively, while $k, T_{e}, T_{0}$, and $\tau_{R}$ are the Boltzmann constant, the temperature of the band electrons, the room temperature, and the electron-temperature relaxation time. Finally, $\Delta E$ is the difference between the energy of the band and that of the traps, and $c=2.5 \times 10^{-4}$ is the ratio between the density of states of the traps and that of the band. Eq. (1) is the transport equation describing the drift motion of the band electrons under the influence of the field, while (2) expresses the power balance of the band electrons: specifically, the $J F$ product at the left hand side provides the power per unit volume injected by the field: such a power is absorbed in part by the phonons (first term at the right hand side), while the other part determines the time change of the band population (second term). Finally, (3) relates the fraction $n_{B} / n$ of the electrons that belong to the band to the electrons' temperature.

The three model equations (1,2,3) involve four unknowns, namely, $J, F, n_{B}$, and $T_{e}$. In a steady-state condition, the equations reduce to a single, algebraic relation involving $F$ and $J$ only. However, the use of the $F, J$ variables is awkward, and it is found that the adoption of auxiliary variables is more suitable because it provides expressions that are easily managed analytically without introducing approximations. This is true also for the time-dependent analysis. To proceed along this line one defines the normalization parameters

$$
a=\frac{\Delta E}{k T_{0}}=14, \quad F_{0}^{2}=\frac{k T_{0}}{q \mu \tau_{R}}=10^{10} \frac{\mathrm{V}^{2}}{\mathrm{~cm}^{2}},
$$

with $\Delta E=360 \mathrm{meV}, \tau_{R}=1 \mathrm{ps}, \mu=2.58 \mathrm{~cm}^{2} /(\mathrm{V} \mathrm{s})$ [16], along with $q=1.6 \times 10^{-19} \mathrm{C}, k=1.38 \times 10^{-23} \mathrm{~J} \mathrm{~K}^{-1}$, and $T_{0}=300 \mathrm{~K}$. Using the auxiliary variables

$$
r=\frac{T_{e}}{T_{0}}, \quad f=\frac{F^{2}}{F_{0}^{2}},
$$

with $T_{0}=300 \mathrm{~K}$, reduces the steady-state form of (2) to

$$
f=(r-1)[1+c \exp (a / r)] .
$$

The graph of (6) is shown in Fig. 1 along with the straight line $f=f_{u}=2.6$, corresponding to $F \simeq 1.6 \times 10^{5} \mathrm{~V} / \mathrm{cm}$. The $f=f_{u}=2.6$ line has been chosen by way of example. In fact, it intersects the $f(r)$ curve in three points; in particular, the intersection at $r_{u}=1.4$ belongs to the negative-slope branch 


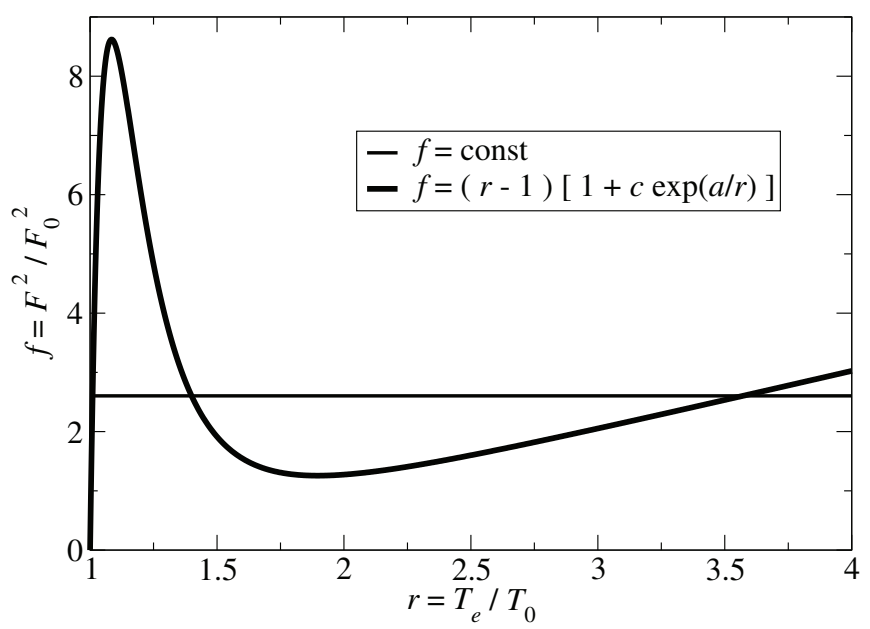

Fig. 1. The thick line shows the graph of $f=(r-1)[1+c \exp (a / r)]$. It is intersected by the thin line $f=f_{u}=2.6$ in three equilibrium points; of them, the one corresponding to $r_{u}=1.4$ intersects the $f(r)$ curve in the negative-slope branch. The form of the expressions used here implies that $r$ is the independent variable, so that the device is "temperature driven" and is loaded at a constant field.

of $f(r)$. The form of the expressions used here implies that $r$ is the independent variable, so that the device is "temperature driven" and is loaded at a constant field.

\section{Stability Analysis}

\section{A. Constant-Field Case}

To determine the stability of the intersection points one resorts to the time-dependent model $(1,2,3)$. As the two intersections with the positive-slope branches are stable, the only equilibrium point considered from now on is that corresponding to $r=r_{u}$. Using the symbols previously defined, and letting $\theta=t / \tau_{R}$, transforms (2) into

$$
\frac{\mathrm{d} r}{\mathrm{~d} \theta}=A(r, f)=\frac{N(r, f)}{D(r)}
$$

with

$$
N(r, f)=(r-1) \frac{n}{n_{B}}-f, \quad D(r)=\frac{a^{2}}{r^{2}}\left(\frac{n_{B}}{n}-1\right) .
$$

Note that in time-dependent conditions (6) does not hold, so the right hand side of (7) does not vanish, apart from the equilibrium point where $N_{u}=N\left(r_{u}, f_{u}\right)=0$. Eq. (7) must be coupled with the differential equation for $f$, that reads $\mathrm{d} f / \mathrm{d} \theta=0$. These two equations describe the dynamic behavior of the system. The analysis then proceeds in the standard way [18], namely, by examining the behavior of the system near the equilibrium point; one lets $r=r_{u}+\delta r, f=f_{u}$ and linearizes (7) about $\left(r_{u}, f_{u}\right)$, to find

$$
\frac{\mathrm{d}}{\mathrm{d} \theta} \delta r=s_{r u} \delta r, \quad s_{r u}=\left(\frac{\partial A}{\partial r}\right)_{u},
$$

whence

$$
\delta r=\delta r_{t=0} \exp \left(s_{r u} \theta\right) .
$$

It follows that the equilibrium point is stable (unstable) if $s_{r u}<0\left(s_{r u}>0\right)$. Remembering (7) one finds

$$
s_{r u}=\frac{D_{u} N_{u}^{\prime}-N_{u} D_{u}^{\prime}}{D_{u}^{2}}
$$

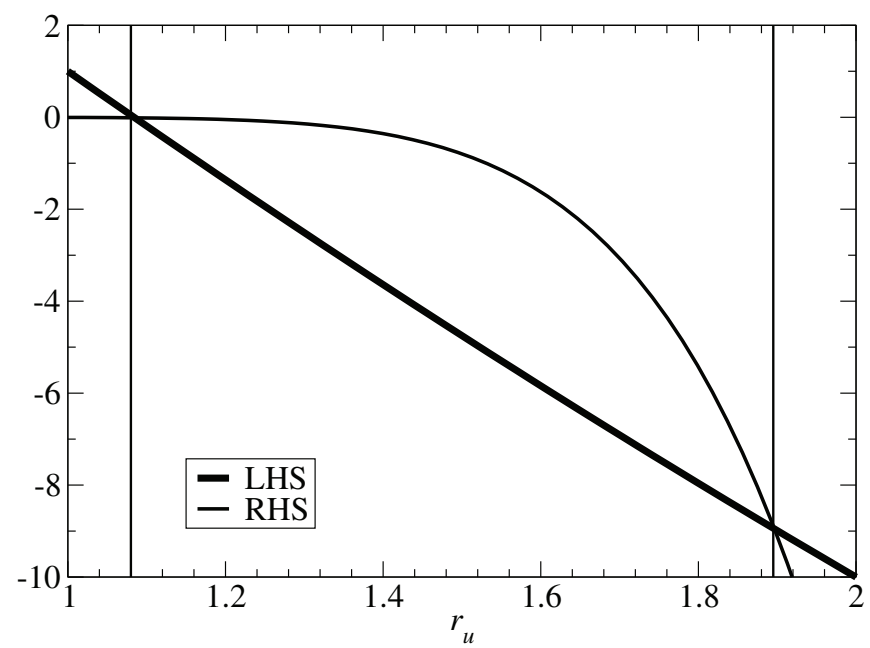

Fig. 2. Graphic solution of (14). The thick (thin) line is the graph of the left (right) hand side. There are two solutions $r_{u 1}, r_{u 2}$, indicated by the vertical lines.

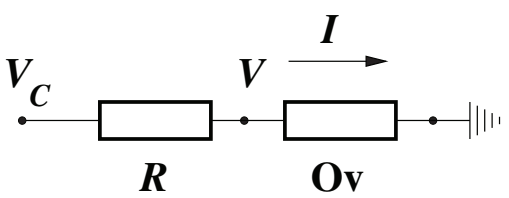

Fig. 3. The bipole obtained by connecting a series resistance $R$ to the Ovonic device (Ov). The position of the load characteristic, and its slope at the intersection with the Ovonic characteristic, is controlled by $R$ and $V_{C}$ or by other two parameters connected to them (like, e.g., $f_{C}$ and $\omega$ defined in the text).

$$
N_{u}^{\prime}=\left(\frac{\partial N}{\partial r}\right)_{u}, \quad D_{u}^{\prime}=\left(\frac{\mathrm{d} D}{\mathrm{~d} r}\right)_{u},
$$

where it is $N_{u}=0$ and $D_{u}=(a / r)^{2}\left(n_{B} / n-1\right)<0$ for all $r$. As a consequence, $s_{r u}=-N_{u}^{\prime} /\left|D_{u}\right|$, and stability is dictated by the sign of $N_{u}^{\prime}$. On the other hand it is

$$
N_{u}^{\prime}=\frac{n}{n_{B}}-a \frac{r-1}{r^{2}}\left(\frac{n}{n_{B}}-1\right),
$$

which may be either positive or negative. It follows that the limiting situation between stability and instability holds for $N_{u}^{\prime}=0$, namely, from (1) and (3),

$$
r_{u}^{2}-a\left(r_{u}-1\right)=-\frac{r_{u}^{2}}{c} \exp \left(-a / r_{u}\right) .
$$

The graphic solution of (14) is shown in Fig. 2. There are two solutions $r_{u 1}, r_{u 2}$, indicated by the vertical lines. In the interval of $r_{u}$ between the solutions the right hand side of (14) is larger than the left hand side, which is equivalent to $N_{u}^{\prime}<0$ and $s_{r u}=N_{u}^{\prime} / D_{u}>0$; as a consequence, the solution at $r_{u}=1.4$ is unstable. Also, from the first of (8) and (6) one notes that $\partial N / \partial r=0$ is identical to $\mathrm{d} f / \mathrm{d} r=0$, so that the points $r_{u 1}$, $r_{u 2}$ are the boundaries of the negative-slope branch. It follows, as expected, that for a load of the type $f=$ const, equilibrium is unstable at all points of the negative-slope branch.

\section{B. Variable-Field Case}

The stability analysis is now repeated in the more general case where the field is allowed to change. To this purpose one 


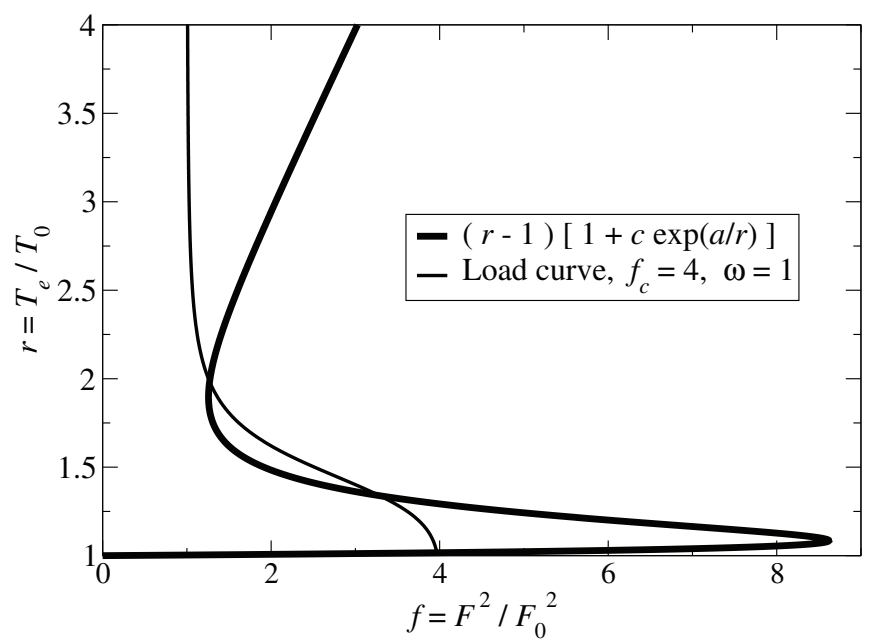

Fig. 4. The thick line shows the graph of the inverse function of $f=$ $(r-1)[1+c \exp (a / r)]$. The thin line shows the graph of the inverse function of (16), with $f_{C}=4, \omega=1$. The two lines intersect each other in three equilibrium points; of them, the one corresponding to $f_{u} \simeq 3.3$ belongs to the negative-slope branch, and is unstable.

connects a series resistance $R$ to the Ovonic device and applies a voltage $V_{C}$ to the bipole thus obtained, so that $V_{C}=V+R I$ (Fig. 3); in the above, $V=L F$ is the voltage drop across the Ovonic device, with $L$ its length, and $I=A J$ the current through it, with $A$ its cross-sectional area. This setup provides more degrees of freedom to the system and eventually yields the required information about the stability of the measurement scheme. As before, the sample under investigation is assumed to be one dimensional and uniform, hence the equations bear no dependence on position. If $L_{R}, A_{R}$ are the resistor's length and cross-sectional area, and $\varrho=R A_{R} / L_{R}$ the resistivity of the resistor's material, letting $\lambda=L / L_{R}$ and $\alpha=A / A_{R}$ one obtains $V_{C} / L_{R}=\lambda F+\alpha \varrho J$. Taking $J$ from (1) and defining the dimensionless parameters $m=\varrho q \mu n, \omega=m \alpha / \lambda$ yields

$$
\frac{F}{F_{C}}=\frac{1+c \exp (a / r)}{\omega+1+c \exp (a / r)}, \quad F_{C}=\frac{V_{C}}{L} .
$$

The original setup with $R=0$ is obtained by letting $\omega \rightarrow 0$ in (15). Finally, squaring the first of (15) and letting $f_{C}=$ $F_{C}^{2} / F_{0}^{2}$, one obtains the load curve

$$
\frac{f}{f_{C}}=\left[\frac{1+c \exp (a / r)}{\omega+1+c \exp (a / r)}\right]^{2} \text {. }
$$

The load curve (16) is shown in Fig. 4 using the values $V_{C}=$ $400 \mathrm{mV}, L=20 \mathrm{~nm}$ (whence $f_{C}=4$ ), and $\omega=1$. Note that, despite the use of a linear component $R$ in the bias circuit, the load curve is not a straight line; this is simply due to the choice of the auxiliary variables made at the beginning, and does not influence the stabillty analysis carried out here.

The dynamic properties are now investigated by linearizing (7) and

$$
B(r, f)=\frac{f}{f_{C}}-\left[\frac{1+c \exp (a / r)}{\omega+1+c \exp (a / r)}\right]^{2}=0
$$

around $r_{u}, f_{u}$; when this is done, both $r$ and $f$ vary. Thus,

$$
\frac{\mathrm{d}}{\mathrm{d} \theta} \delta r=s_{r u} \delta r-\frac{\delta f}{D_{u}}, \quad w_{r u} \delta r+\frac{\delta f}{f_{C}}=0,
$$

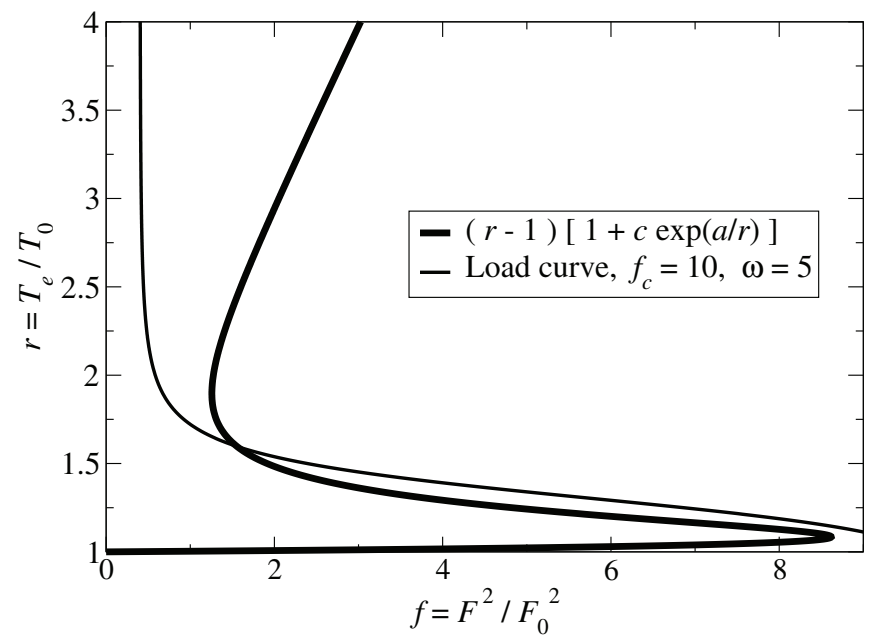

Fig. 5. The thick line shows the graph of the inverse function of $f=$ $(r-1)[1+c \exp (a / r)]$. The thin line shows the graph of the inverse function of (16), with $f_{C}=10, \omega=5$. The two lines intersect each other in one equilibrium point only, that belongs to the negative-slope branch, and is stable.

$$
s_{r u}=\frac{N_{u}^{\prime}}{D_{u}}, \quad w_{r u}=-\frac{\mathrm{d}}{\mathrm{d} r}\left[\frac{1+c \exp (a / r)}{\omega+1+c \exp (a / r)}\right]_{u}^{2} .
$$

Eliminating $\delta f$ from (18) provides

$$
\frac{\mathrm{d}}{\mathrm{d} \theta} \delta r=\frac{N_{u}^{\prime}+f_{C} w_{r u}}{D_{u}} \delta r
$$

whence, remembering that $D_{u}<0$, the equilibrium point is stable (unstable) if $N_{u}^{\prime}+f_{C} w_{r u}>0\left(N_{u}^{\prime}+f_{C} w_{r u}<0\right)$. It has been shown before that $N_{u}^{\prime}<0$ at all points of the negative-slope branch; on the other hand it is

$$
w_{r u}=\frac{2 \omega a / r_{u}^{2}}{\left(\omega+n / n_{B}\right)^{3}}\left(\frac{n}{n_{B}}-1\right) \frac{n}{n_{B}}>0,
$$

showing that the sign of $N_{u}^{\prime}+f_{C} w_{r u}$ depends on the choice of the two parameters $f_{C}$ and $\omega$. Replacing $N_{u}^{\prime}$ with $\mathrm{d} f / \mathrm{d} r$ and observing that $f_{C} w_{r u}$ is the negative derivative of the load curve (16) with respect to $r$, one finds that the stability condition is summarized as

$$
\left|\frac{\mathrm{d} f}{\mathrm{~d} r}\right|_{\text {load }}>\left|\frac{\mathrm{d} f}{\mathrm{~d} r}\right|_{\text {Ov }} .
$$

For instance, the point corresponding to $f_{u} \simeq 3.3$ in Fig. 4 is unstable, whereas the only intersection between the Ovonic and load curves in Fig. 5 is stable (note that the slopes must be appreciated by wieving from the $r$ axis). The value $f_{C}=12$ used in Fig. 5 corresponds to $V_{C} \simeq 630 \mathrm{mV}$.

\section{CONCLUSions}

In summary, using the parameters of the generalized model $(7,16)$ it is possible to fix the load curve of the Ovonic device in such a way that the intersects in the negative-slope branch become stable. Thus, suitable combinations of $V_{C}$ and $R$ make it possible to sweep the whole branch, which is equivalent to making the measurement of the complete $I(V)$ curve feasible.

The theory upon which the analysis of this paper is based assumes that the time variation of the unknowns is due only to 


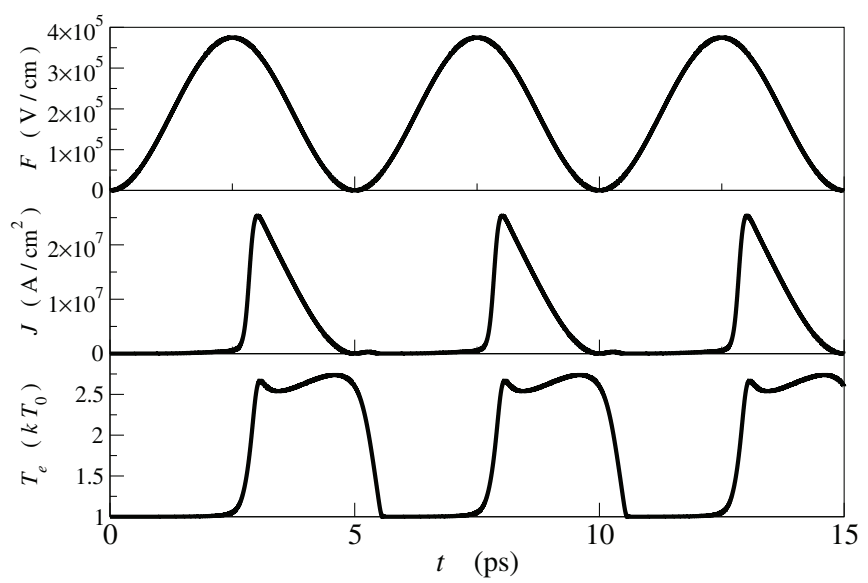

Fig. 6. Outcome of the dynamic model when $R=0$ and $V_{C}=(3 / 4)[1-$ $\cos (2 \pi t / T)] \mathrm{V}$, with $T=5 \mathrm{ps}$. The graph of the current density $J$ and carrier temperature $T_{e}$ with respect to time are shown in the middle and lower part of the figure, respectively.

the last term of (2), namely, it is intrinsic to the device. In other words, parasitic effects belonging to the measuring apparatus are not considered. In practical situations such effects are present, and can be described by inserting, e.g., an external capacitance $C$ in parallel to the Ovonic device. This introduces another time constant $\tau=R C$, to be compared with the intrinsic time constant given by the interplay of $\tau_{R}$ and $\Delta E$ in (2). However, the analysis of stability is carried out along the same line of reasoning, albeit the quantitative results are affected by the difference in magnitude of the two time constants.

The time-dependent model $(1,2,3)$ can also be used to analyze the behavior of an Ovonic device subjected to an oscillatory bias. As the $I(V)$ characteristic of the device is non linear, such a calculation must be carried out numerically; from a qualitative viewpoint, if the bias depends linearly on, say, $\cos (2 \pi \nu t)$, the internal variables are expected to oscillate with the same frequency $\nu$, albeit their form may strongly depart from the sinusoidal one. This is in fact confirmed by Fig. 6, that shows the result of a simulation carried out by letting $R=0$ and applying a voltage of the form

$$
V_{C}=\frac{3}{4}\left[1-\cos \left(2 \pi \frac{t}{T}\right)\right] \quad(\mathrm{V}),
$$

with $T=5 \mathrm{ps}$, corresponding to $\nu=200 \mathrm{GHz}$. From $R=0$ it follows that, when the auxiliary variables $r, f$ are used, the load curve is of the type shown in Fig. 1. The length of the Ovonic device used in the simulation is $L=40 \mathrm{~nm}$; as a consequence, the electric field $F$ derived from (23) ranges between 0 and $3.75 \times 10^{5} \mathrm{~V} / \mathrm{cm}$. The upper part of Fig. 6 shows the time dependence of the electric field $F$; in turn, the middle and lower parts show, respectively, the time dependence of the current density $J$ and electron temperature $T_{e}$. As expected, the period of $J$ and $T_{e}$ is the same as that of the input signal $F$, although the waveforms are significantly distorted with respect to the sinusoidal form.

\section{REFERENCES}

[1] S. R. Ovshinsky, "Reversible electrical switching phenomena in disordered structures," Phys. Rev. Lett., vol. 21, p. 1450, 1968.

[2] D. Adler, "Switching phenomena in thin films," J. Vac. Sci. Technol., vol. 10, p. 728, 1973.

[3] D. Adler, M. S. Shur, M. Silver, and S. R. Ovshinsky, "Threshold switching in chalcogenide-glass thin films," J. Appl. Phys., vol. 51, p. 3289, 1980.

[4] M. Nardone, V. G. Karpov, D. C. S. Jackson, and I. V. Karpov, "A unified model of nucleation switching," Appl. Phys. Lett., vol. 94, p. 103509, 2009.

[5] M. Simon, M. Nardone, V. G. Karpov, and I. V. Karpov, "Conductive path formation in glasses of phase change memory," J. Appl. Phys., vol. 108, p. 064514, 2010.

[6] A. Pirovano, A. Lacaita, A. Benvenuti, F. Pellizzer, and R. Bez, "Electronic switching in phase-change memories," IEEE Trans. Electron Devices, vol. 51, p. 452, 2004.

[7] D. Ielmini and Y. Zhang, "Analytical model for subthreshold conduction and threshold switching in chalcogenide-based memory devices," $J$. Appl. Phys., no. 102, p. 054517, 2007.

[8] D. Ielmini, "Threshold switching mechanism by high-field energy gain in the hopping transport of chalcogenide glasses," Phys. Rev. B, vol. 78, p. $035308,2008$.

[9] M. Rudan, F. Giovanardi, E. Piccinini, F. Buscemi, R. Brunetti, and C. Jacoboni, "Voltage snapback in amorphous-GST memory devices: Transport model and validation," IEEE Trans. Electron Devices, vol. 58, p. 4361, 2011.

[10] E. Piccinini, A. Cappelli, F. Buscemi, R. Brunetti, D. Ielmini, M. Rudan, and C. Jacoboni, "Hot-carrier trap-limited transport in switching chalcogenides," J. Appl. Phys., vol. 112, p. 083722, 2012.

[11] A. Cappelli, E. Piccinini, F. Xiong, A. Behnam, R. Brunetti, M. Rudan, E. Pop, and C. Jacoboni, "Conductive preferential paths of hot carriers in amorphous phase-change materials," Appl. Phys. Lett., vol. 103, p. 083503, 2013 .

[12] S. Raoux and M. Wuttig, Phase Change Materials: Science and Applications. Springer, 2010.

[13] S. Lavizzari, D. Ielmini, D. Sharma, and A. Lacaita, "Transient effects of delay, switching and recovery in phase change memory (PCM) devices," in Proc. International Electron Devices Meeting (IEDM). San Francisco: IEEE, 2008, pp. 1-4.

[14] S. Lavizzari, D. Sharma, and D. Ielmini, "Threshold-switching delay controlled by $1 / f$ current fluctuations in phase-change memory devices," IEEE Trans. Electron Devices, vol. 57, p. 1047, 2010.

[15] S. Lavizzari, D. Ielmini, and A. Lacaita, "Transient simulation of delay and switching effects in phase-change memories," IEEE Trans. Electron Devices, vol. 57, p. 3257, 2010.

[16] F. Buscemi, E. Piccinini, A. Cappelli, R. Brunetti, M. Rudan, and C. Jacoboni, "Electrical bistability in amorphous semiconductors: a basic analytical theory," Appl. Phys. Lett., vol. 104, p. 022101, 2014.

[17] F. Buscemi, E. Piccinini, R. Brunetti, M. Rudan, and C. Jacoboni, "Time-dependent transport in amorphous semiconductors: Instability in the field-controlled regime," Appl. Phys. Lett., vol. 104, p. 262106, 2014.

[18] A. M. Lyapounov, "Problème Général de la Stabilité du Mouvement," Ann. Fac. Sc. Univ. Toulouse, vol. 9, no. 2, pp. 203-475, 1907 (in French). 